\title{
Schlieren Methods for High Pressure Turbulent Jet Flames
}

\author{
$\mathrm{Li} \mathrm{Li}^{1} *$ Fu Shan ${ }^{2} \& \mathrm{~J} \mathrm{~J}$ Witton ${ }^{3}$
}

\section{Summary}

Turbulent flame speeds of a pre-mixed synthetic natural gas mixture were measured using a jet-flow apparatus over a range of pressures to $0.8 \mathrm{MPa}$ at an equivalence ratio of 0.9. The method adopted was the 'flame angle' technique, using schlieren imaging to obtain the flame vertex angle from the peak density gradient. Image analysis techniques were developed to reduce interpretation errors and to give information on the velocity and combined gradient distribution local to the flame front.

Key words: Flame speed; Schlieren; Optical methods; Image analysis; Combustion

${ }^{1}$ Combustor \& Heat Transfer Technology Group, now at RWE NPower Company, li.li@rwenpower.com

${ }^{2}$ Image Processing Group, now at Shanghai JiaoTong University, sfu@ sjtu.edu.cn

${ }^{3}$ Combustor \& Heat Transfer Technology Group, School of Engineering, Cranfield University, Bedford, MK43 0AL, j.j.witton@cranfield.ac.uk

* Corresponding Author 


\section{Introduction}

The main subject of the work relates to high efficiency industrial gas turbines, where stabilization of lean combustion is critical to engine emission performance and durability. The turbulent flame speed, $\mathrm{S}_{\mathrm{T}}$, characterizes the mass rate of reactant consumption per unit area of the flame surface and plays an important role in formulating predictive, reliable, and robust models of turbulent combustion for multidimensional simulations of flames. Flame speed varies with reactant pressure and temperature and can be measured by several different techniques.

\section{Test Method}

Our measurements have been made with a tube-stabilized conical (Bunsen) flame, using the angle method $\left(S_{T}=U \sin (\theta / 2)\right.$, where $U$ is the mean velocity of mixture at the tube outlet and $\theta$ is the vertex angle of the mean flame region). However the determination of $S_{\mathrm{T}}$ is difficult as the flame front is oblique and the thickness of the flame brush increases with increasing distance from the stabilization region.

Most premixed turbulent flames of practical interest are within the flamelet regime. Thin flamelets also mean that the flame zone is characterized by large density gradients. This offers high sensitivity to the application of light deflection techniques such as schlieren, deflection mapping and interferometry. A typical flamelet oriented parallel to the incident beam direction produces a density gradient orthogonal to beam and because schlieren is only sensitive to density gradients orthogonal to the beam, flamelets normal to the beam direction will be transparent.

For the conical flame configuration, schlieren can outline the flame cone very clearly. ${ }^{1}$ The schlieren method is simple and avoids some of the difficulties associated with operation of the flame at elevated pressure, such as the pressure-broadening effects for flame brush thickness. Schlieren uses density gradient imaging with natural light emission. The basic principle of the technique is the combination of the optical projection of a flow perturbation with an indication of its light deflection. The method translates phase differences into visible-spectrum amplitude and colour differences. The optics are relatively simple and do not require a high power laser source or very precise alignment. Although it is a so-called 'line-of-sight integration' technique, it has been shown that with the selection of the proper flame configuration to take advantage of the unique features of premixed flames, quantitative information can be deduced from the schlieren images. ${ }^{2-3}$

Several physical mechanisms can contribute to the dependence of the value of $S_{T}$ on the choice of a flame surface and on the flame geometry, such as the unsteadiness of the mean flame brush thickness, the changes of the normal convective mass flux across the flame and the curvature of the mean flame brush. Also, because experimental determination of the turbulent burning velocity usually requires defining a flame surface to represent the thick turbulent flame brush in addition to measuring the mean velocity entering the flame, there exist uncertainties of determining the flame velocity.

These measurement uncertainties are reduced by an average of a number of schlieren images and carefully designed data processing. 


\section{The Schlieren System}

The optical system was built around a folded-path arrangement using large spherical mirrors, diameter $305 \mathrm{~mm}$, to reduce distortion. Images were generated using a Xenon flash lamp source (100hz repetition rate, $60 \mathrm{~W}$ average input, 2.90us average flash duration) driven by a signal generator and CCD camera $(1024 \times 1260$ resolution) to capture the images. Image files were dumped directly to a PC hard drive. The window apertures were $100 \mathrm{~mm}$ and the whole window area could be imaged.

Effects of flame radiation have been controlled using a filter in front of the camera lens and short camera shutter duration $(1 \mathrm{~ms})$. The original instantaneous images were taken from the CCD, and averaged image was determined by an average of pixel data taken from fifty images. A program was written to find the digital profile of flame front mainly according to the brightness of the edge, assuming axisymmetry of the flame. In the program, the flame zone is firstly chosen from the averaged image, the threshold value is determined considering the histogram and average pixel data for each scan line.

\section{Data Processing}

Flow visualization can determine the flame front located at the sharp boundary between light and dark regions representing the maximum temperature gradient. Given the regime of premixed turbulent combustion, it also marks the regions of unburned and burned gases.

Flow visualization has the advantages of showing the spatial realization of the dynamic evolution simultaneously; however it can only provide qualitative observation. Fu. ${ }^{4}$ proposed that the "image flow field" of the schlieren images could represent the fluid flow field, and the velocity distribution could then be recovered using image motion detection. This data processing method can both give visualization and provide the prospects of the instantaneous measurement.

For this processing method, instead of using schlieren image sequence, only one instantaneous image was used to recover the velocity distribution: here one image is separated into two consecutive ones according to odd and even lines, the velocity gradients $\left(\left|\frac{d u}{d x}\right|,\left|\frac{d u}{d y}\right|,\left|\frac{d v}{d x}\right|,\left|\frac{d v}{d y}\right|\right)$ are calculated to determine the flame front. Moreover the combined gradient maps (CGM, $\left|\frac{d u}{d x}\right|+\left|\frac{d u}{d y}\right|+\left|\frac{d v}{d x}\right|+\left|\frac{d v}{d y}\right|$ ) of flow is studied and compared with the flame front.

\section{Case Study}

Here we selected three conditions of different pressures, both instantaneous and averaged schlieren images are shown with derived flame envelope over a range of conditions. In this study, averaged image is got from 50 instantaneous images. 


\begin{tabular}{|c|c|c|c|c|c|c|c|c|c|c|c|c|}
\hline & $\begin{array}{c}\mathbf{P} \\
(\mathbf{k P a})\end{array}$ & $\begin{array}{c}\mathrm{V} \\
\left(\mathrm{m} \cdot \mathrm{s}^{-1}\right)\end{array}$ & $\begin{array}{c}\mathbf{u}^{\prime} \\
\left.\mathbf{m} . \mathrm{s}^{-1}\right)\end{array}$ & $\begin{array}{c}\mathrm{Tu} \\
(\%)\end{array}$ & $\frac{\mathrm{S}_{\mathrm{L}}}{\left(\mathrm{m}_{\mathrm{s}} \mathbf{s}^{-1}\right)}$ & $\frac{\mathbf{S}_{\mathrm{T}}}{\left(\mathbf{m}^{-\mathbf{s}^{-1}}\right)}$ & $\begin{array}{c}\delta \\
(\mathbf{m m})\end{array}$ & $\begin{array}{c}\boldsymbol{\eta} \\
(\mathbf{m m})\end{array}$ & $\begin{array}{c}\mathbf{I}_{\mathbf{t}} \\
(\mathbf{m m})\end{array}$ & $\mathbf{R e}_{\mathrm{T}}$ & Da & Ka \\
\hline Case1 & 100 & 4.32 & 0.2 & 4.89 & 0.3436 & 0.765 & 0.065 & 0.224 & 4.98 & 62.64 & 132 & 0.084 \\
\hline Case2 & 300 & 4.05 & 0.262 & 5.94 & 0.2833 & 1.47 & $\mathbf{0 . 0 3 4}$ & $\mathbf{0 . 1 3 5}$ & 18.42 & 703.5 & 586 & 0.063 \\
\hline Case3 & 700 & 7.4 & 0.363 & 4.69 & 0.1982 & $\mathbf{3 . 3 0 9}$ & $\mathbf{0 . 0 2 2}$ & 0.06 & 22.4 & 2665 & 556 & 0.134 \\
\hline
\end{tabular}

Where: $\mathrm{P}=$ mixture pressure; $\mathrm{V}=$ nozzle velocity; $\mathrm{u}^{\prime}=$ fluctuating velocity; $\mathrm{Tu}=$ turbulence intensity;

$\mathrm{S}_{\mathrm{L}}=$ laminar flame speed calculated from CHEMKIN; $\mathrm{S}_{\mathrm{T}}=$ measured turbulent flame velocity;

$\delta=$ flame thickness $=\left(\lambda_{\mathrm{u}} /\left(\rho_{\mathrm{u}} \mathrm{Cp}_{\mathrm{u}} \mathrm{S}_{\mathrm{L}}\right)\right.$ (for u: unburnt conditions); $\eta=$ Kolmogoroff scale $\left(\mathrm{It} / \operatorname{Re}_{\mathrm{T}}{ }^{3 / 4}\right) ;$

$\mathrm{I}_{\mathrm{t}}=$ Integral length scale; $\operatorname{Re}_{\mathrm{T}}=$ nozzle Reynolds $\mathrm{N}^{\mathrm{o}}=\left(\mathrm{u}^{\prime} \mathrm{lt} / \delta / \mathrm{S}_{\mathrm{L}}\right) ; \mathrm{Da}$, Damköhler $\mathrm{N}^{\mathrm{o}}=\left(\mathrm{lt} \mathrm{S}_{\mathrm{L}} / \delta / \mathrm{u}^{\prime}\right)$;

Ka, Karlovitz $N^{0}=\left(\operatorname{Re}_{\mathrm{T}}^{-1 / 2}\left(\mathrm{u}^{\prime} / \mathrm{S}_{\mathrm{L}}\right)^{2}\right)$

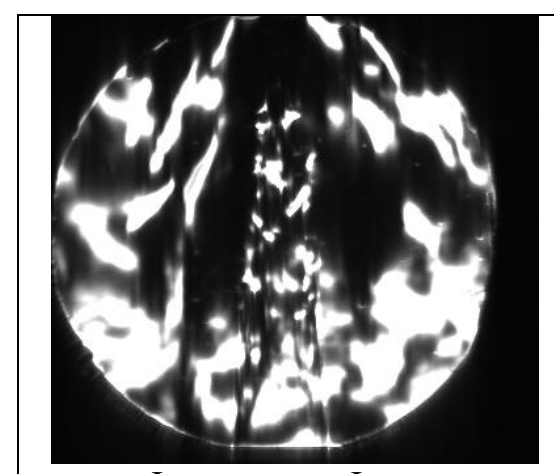

Instantaneous Image

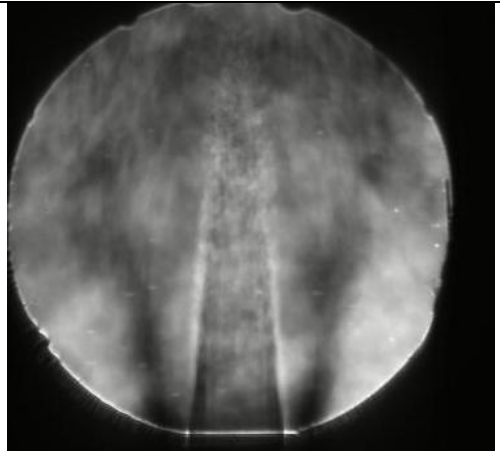

Averaged Image Case 1

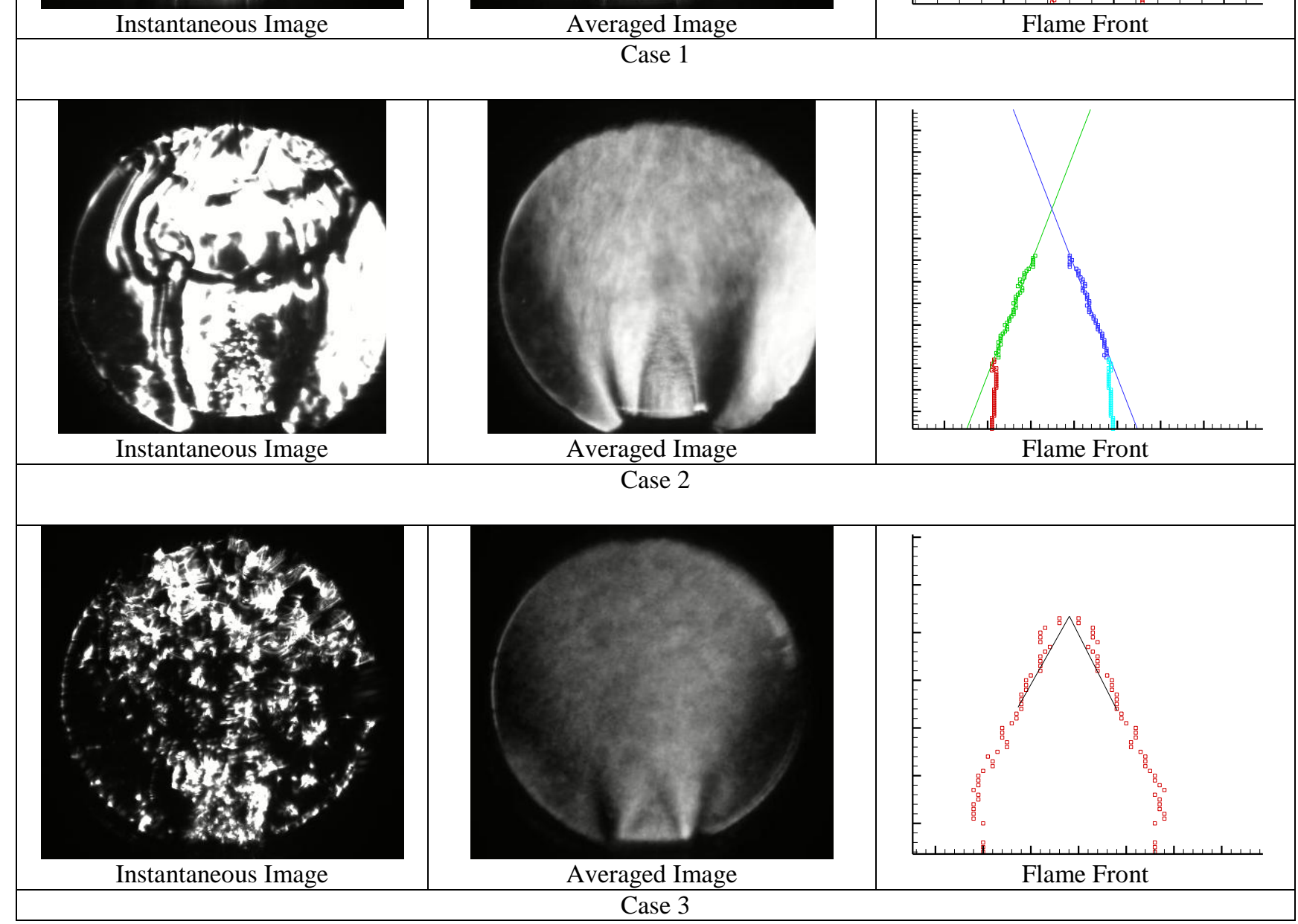

Fig. 1 Schlieren Image Data Processing 


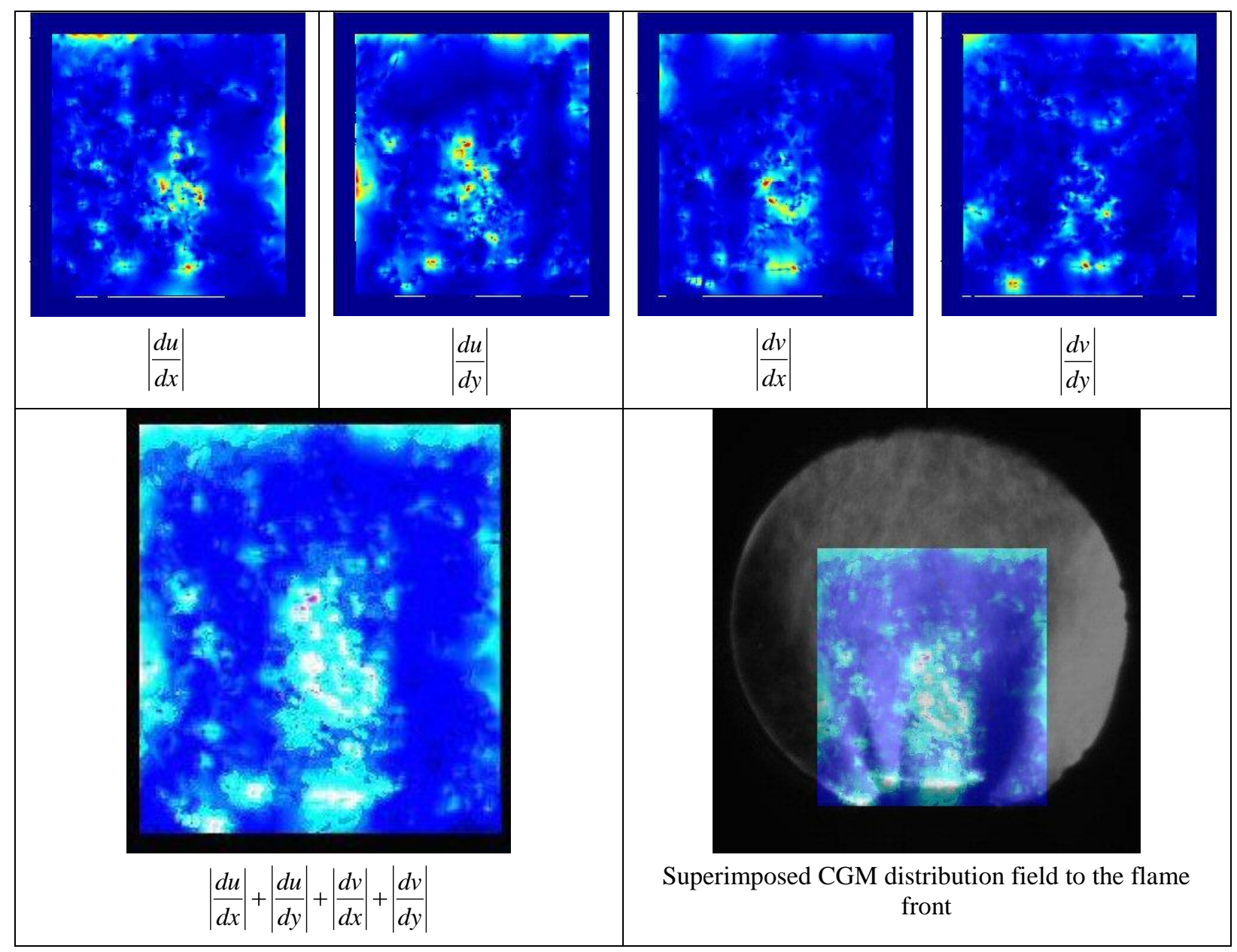

Fig. 2 CGM distribution field for averaged image of Case 2
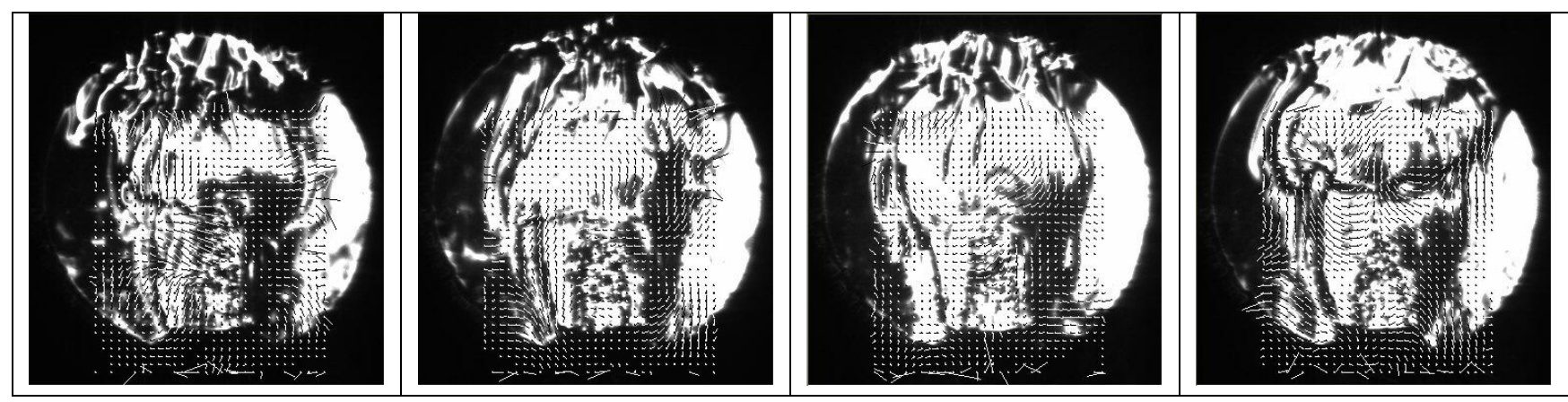

Fig. 3 Superimposed velocity distribution field to the instantaneous images of case 2

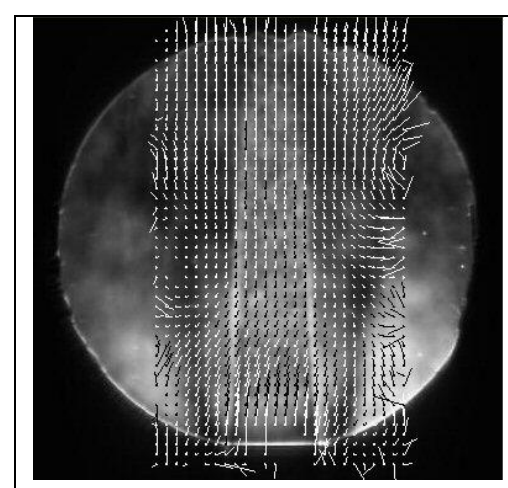

Case 1

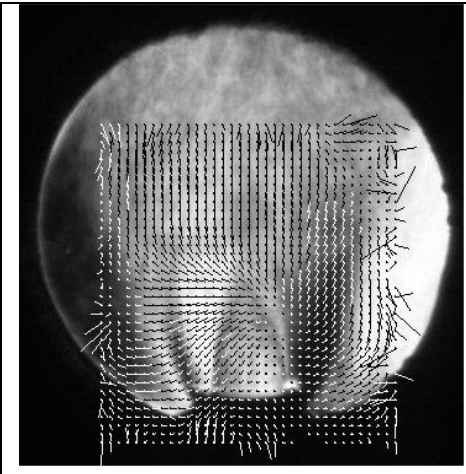

Case 2

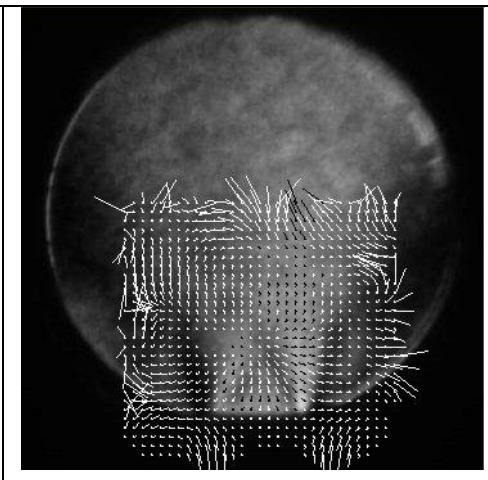

Case 3

Fig. 4 Superimposed velocity distribution field to the averaged image 
At elevated pressure, the flame brush became shorter and less well-defined, schlieren images from the elevated pressure experiments illustrate the difficulty of data manipulation to arrive at flame speed (as shown in Fig.1).

CGM data processing method is illustrated in Fig.2. The fact that the CGM distribution almost overlaps the digital profile drawn from digital image processing shows these two methods result the same flame front (as shown in Figs 3 \& 4).

\section{Discussion and Conclusions}

From schlieren images, the authors can get the flame front, flame speed, velocity and combined gradient distribution field. The instantaneous and average velocity distributions show the fluctuation and statistic property of the flame, the authors can even estimate the velocity quantitatively with further calculation. The fact that the CGM distribution almost overlaps the digital profile drawn from digital image processing shows these two methods result the same flame front.

Schlieren method is very valuable study high pressure turbulent flames, it can supply much information including the flame front, flame speed and velocity distribution.

\section{Acknowledgements}

The flame speed work formed part of a European Commission Framework V project, completed under EC Contract No. ENK5-CT-2000-00060. One author (LL) acknowledges support under this program. The image acquisition and analysis was performed as part of the on-going research of both research groups in the School of Engineering.

\section{References}

1. L.W Kostuik and R.K Cheng: Experiments in Fluids, 1994, 18. 59-68.

2. H. Kobayashi: Combustion and Flame, 1997, 108, 104-117.

3. H. Kobayashi: Twenty-sixth Symposium (International) on Combustion, Naples, Italy, The Combustion Institute 1996, 389-39.

4. S Fu and Y Wu: Optical. Engineering, 2001, 40, 1661 - 1666. 